\section{Neural production of kynurenic acid in Caenorhabditis elegans requires the AAT-1 transporter}

\author{
Lin Lin, ${ }^{1}$ George A. Lemieux, ${ }^{1}$ \\ Osatohanmwen Jessica Enogieru, ${ }^{2}$ \\ Kathleen M. Giacomini, ${ }^{2}$ and Kaveh Ashrafi ${ }^{1}$
}

${ }^{1}$ Department of Physiology, ${ }^{2}$ Department of Bioengineering and Therapeutic Sciences, University of California at San Francisco, San Francisco, California 94158, USA

Kynurenic acid (KynA) levels link peripheral metabolic status to neural functions including learning and memory. Since neural KynA levels dampen learning capacity, KynA reduction has been proposed as a therapeutic strategy for conditions of cognitive deficit such as neurodegeneration. While KynA is generated locally within the nervous system, its precursor, kynurenine (Kyn), is largely derived from peripheral resources. The mechanisms that import Kyn into the nervous system are poorly understood. Here, we provide genetic, anatomical, biochemical, and behavioral evidence showing that in C. elegans an ortho$\log$ of the human LAT1 transporter, AAT-1, imports Kyn into sites of KynA production.

Supplemental material is available for this article.

Received April 7, 2020; revised version accepted June 24, 2020.

Although long overlooked as mere degradation components of tryptophan, it is becoming increasing clear that kynurenine pathway $(\mathrm{KP})$ metabolites are signaling molecules in multiple organ systems (Schwarcz et al. 2012; Cervenka et al. 2017). One of these metabolites, kynurenic acid (KynA), is an antagonist of glutamatergic receptors, including the $\mathrm{N}$-methyl D-aspartate receptors (NMDARs) (Perkins and Stone 1982), which play fundamental roles in learning capacity across phylogeny (Morris et al. 1986; Kano et al. 2008; Vohra et al. 2017). Reductions in KynA improve learning and memory in species as diverse as mammals and C. elegans, while increased KynA levels cause significant diminishments in cognitive functions (Moroni et al. 1988; Erhardt et al. 2001; Schwarcz et al. 2001; Kepplinger et al. 2005; Potter et al. 2010; Pocivavsek et al. 2011; Vohra et al. 2018). Elevated brain KynA levels are linked to neurodegeneration and psychiatric disorders in humans (Schwarcz et al. 2012). Thus, understanding of the molecular mechanisms that determine brain levels of KynA may have therapeutic potential for neurodegenerative and psychiatric disorders (Schwarcz et al. 2012; Stone and Darlington 2013).

In both C. elegans and mammals, KynA is generated at highly spatially restricted patterns in the nervous system, yet neural KynA levels are influenced by factors that act

[Keywords: transporter; learning; metabolism; kynurenic acid; C. elegans] Corresponding author: kaveh.ashrafi@ucsf.edu

Article published online ahead of print. Article and publication date are online at http://www.genesdev.org/cgi/doi/10.1101/gad.339119.120. distantly. This is because kynurenine pathway genes are not all coexpressed in the same tissues or cells. In both C. elegans and mammals, KynA generation occurs in specific regions of the nervous system due to localized expression of the enzyme kynurenine aminotransferase (NKAT-1 in C. elegans), which catalyzes the conversion of Kyn to KynA (Guidetti et al. 2007; Lemieux et al. 2015; Vohra et al. 2017). Neural sites of KynA generation in C. elegans do not appear to generate Kyn. Tryptophan 2,3 dioxygenase (TDO-2 in C. elegans), the enzyme that brings tryptophan into the kynurenine pathway to generate Kyn, is expressed in peripheral tissues, mostly in the skin-like epidermal cells and not in the neurons that express NKAT-1 (van der Goot et al. 2012; Vohra et al. 2017). Thus, there must be mechanisms that allow for the import of Kyn into neural sites of KynA generation. Similarly, the majority of Kyn in the mammalian brain is derived from peripheral sources (Gál and Sherman 1980; Fukui et al. 1991). Processes such as exercise, inflammation, and stress alter peripheral Kyn production with subsequent effects on neural KynA levels and cognitive functions (Agudelo et al. 2014; Cervenka et al. 2017; O'Farrell and Harkin 2017). The mechanisms that allow for the import of Kyn into the nervous system are poorly understood.

Here, we show that AAT-1, a C. elegans counterpart of LAT1, is required for the import of Kyn into specific nkat1-expressing neurons for KynA generation.

\section{Results and Discussion}

Loss of aat-1 increases feeding rate dependent on serotonin signaling

The insight into aat-1 as a component of the kynurenine pathway initially emerged from a screen pertaining to food intake behavior. C. elegans ingest food through pharyngeal pumping, whose rate correlates with food intake (Avery and Horvitz 1990). We found that exposure of $C$. elegans to aat-1 RNA interference (RNAi) results in increased pharyngeal pumping (Fig. 1A; Supplemental Fig. S1). The C. elegans genome contains nine aat family members that potentially encode for the catalytic subunit of a heteromeric amino acid transporter with sequence similarity to mammalian LAT1 (Veljkovic et al. 2004). Other than aat-1 RNAi, only aat-2 RNAi altered pharyngeal pumping rates of C. elegans (Supplemental Fig. S1). Since we did not verify the extent of knockdown in each case, we cannot rule out the possibility that AAT transporters other than AAT-1 and AAT-2 contribute to feeding behavior.

Changes in pumping rate under different nutritional conditions correspond to changes in serotonin signaling (Horvitz et al. 1982; Avery and Horvitz 1990; Sze et al. 2000; Cunningham et al. 2012). Specifically, the reduced pumping rates of animals taken off food is mimicked by loss of tryptophan hydroxylase-1, the rate-limiting

(C) 2020 Lin et al. This article is distributed exclusively by Cold Spring Harbor Laboratory Press for the first six months after the full-issue publication date (see http://genesdev.cshlp.org/site/misc/terms.xhtml). After six months, it is available under a Creative Commons License (Attribution-NonCommercial 4.0 International), as described at http://creativecommons.org/licenses/by-nc/4.0/. 


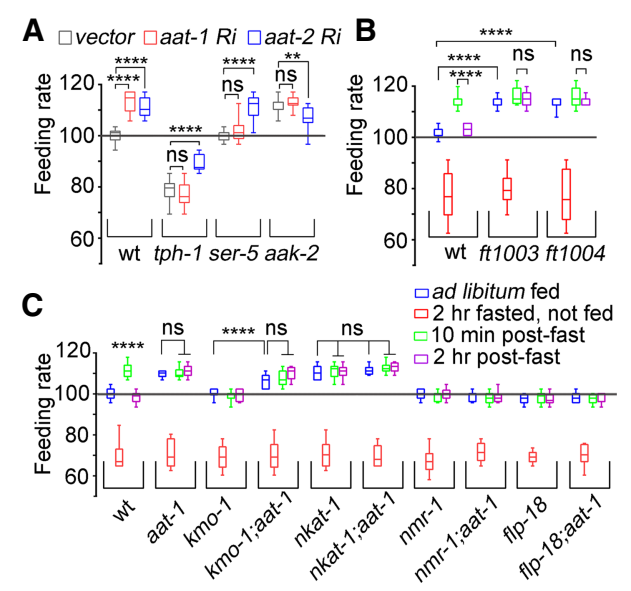

Figure 1. Loss of aat-1 induces hyperactive pharyngeal pumping. (A) Relative pharyngeal pumping rates of wild-type (wt), ser-5, tph1 , and aak-2 mutants cultured on bacteria expressing the indicated RNAi clones $(R i)$. All feeding data are normalized and presented as the percentage of the ad libitum-fed wild-type rates. $n=14$ animals per condition. (ns) $P>0.05$; $\left.{ }^{* *}\right) P<0.01 ;{ }^{(* * *)} P<0.0001$ by ANOVA (Dunnett). (B) Relative pharyngeal pumping rates of wild-type and aat- 1 mutants at the indicated fasting and refeeding periods. ft1003 and ft1004 are putative null alleles of aat-1. $n=12-15$ animals per condition. (ns) $P>0.05$; $\left.{ }^{* * * *}\right) P<0.0001$ by ANOVA (Tukey). (C) Relative pharyngeal pumping rates of wild-type or single or double mutants at the indicated fasting and refeeding periods. $n=10-12$ for each condition. (ns) $P>0.05$; (***) $P<0.0001$ by ANOVA (Tukey). Nonnormalized data for all of the above pharyngeal pumping conditions are in Supplemental Table S1.

enzyme in serotonin biosynthesis, encoded by tph-1 (Sze et al. 2000). In turn, the transiently hyperactivated pumping rates of fasted animals that are returned to food corresponds to transiently hyperelevated serotonin secretion (Lemieux et al. 2015). Well-fed animals are characterized by intermediate levels of serotonin signaling and pumping rate (Lemieux et al. 2015). The feeding effects of hyperelevated serotonin signaling are dependent on signaling by SER-5, a serotonergic G-coupled protein receptor, and subsequent inhibition of AAK-2 containing AMP-activated kinases (Cunningham et al. 2012, 2014). The hyperactive feeding caused by aat-1 deficiency, but not that of aat-2, was dependent on tph-1 and ser-5 (Fig. 1A). Moreover, aat-1 RNAi did not further increase the already elevated pumping rates of aak-2 mutants (Fig. 1A). These results suggested that aat-1 may function upstream of or parallel to the serotonin signaling pathway to regulate feeding.

\section{Loss of AAT-1 mimics the effects of KynA deficiency} on feeding rate

To further study the function of AAT-1 and verify the RNAi results, we generated aat-1 mutants using the CRISPR/Cas9 system and obtained two independent mutations: aat-1 (ft1003) and aat-1(ft1004). Both deletions remove most of the predicted protein coding regions of AAT-1 and thus are likely to be strong loss-of-function or null mutations (Supplemental Fig. S2). Consistent with our RNAi results, aat-1 deletion mutants displayed a constitutively elevated pumping rate (Fig. 1B). These mutants reduced their pharyngeal pumping rates to the same level seen in wild-type animals during the fasted state. Upon exposure to food after fasting, wild-type and aat-1 deletion mutants exhibited hyperactive feeding rates (Fig. 1B). While this hyperactive rate was transient in wild-type animals, the aat-1 mutants maintained the hyperactive feeding rates (Fig. 1B). This pattern was reminiscent of animals deficient in neural KynA levels (Lemieux et al. 2015).

We demonstrated previously that neurally produced KynA serves as the molecular mechanism by which C. elegans assesses its experience of fasting to then allow for the transient hyperelevated serotonin signaling and the resulting hyperactive food intake behavior (Lemieux et al. 2015). Upon fasting, KynA levels deplete in a time dependent manner resulting in enhanced activity of specific NMDAR-expressing neurons (Lemieux et al. 2015; Vohra et al. 2017). This leads to elevated signaling by FLP-18, an FMFR family neuropeptide with functions reminiscent of mammalian neuropeptide Y molecule (Lemieux et al. 2015). FLP-18 signaling to specific serotonergic neurons poises them to hypersecrete serotonin upon exposure to food, which drives the postfast hyperactive feeding (Lemieux et al. 2015). The similar feeding phenotypes of aat-1 and nkat-1 mutants and their similar genetic relationships to serotonin signaling prompted us to examine whether the feeding increasing effects of aat-1 loss are dependent on the same molecular mechanisms that mediate the effects of KynA deficiency. We found this to be the case: The hyperactive feeding of aat-1 mutants was dependent on $n m r-1$ and $f l p-18$ (Fig. 1C). Moreover, as in KynA deficient animals, aat-1 mutants that also lacked either nmr1 or $f l p-18$ failed to display hyperactive pumping rates upon exposure to food after fast (Fig. 1C). Additionally, aat-1;nkat-1 double mutants exhibited hyperactive feeding similar to aat-1 or nkat-1 single mutants and exposure to food after fasting did not result in any additional increases in pumping rates (Fig. 1C). Additional evidence suggesting a role for aat-1 in determining neural levels of KynA emerged from analysis of aat-1;kmo-1 double mutants. We previously observed that $\mathrm{kmo}-1$ null mutants have elevated KynA levels and while they exhibit wild-type feeding rates when well fed, they cannot hyperactive their pumping rates after fast (Lemieux et al. 2015). The elevated neural KynA levels seen in kmo-1 mutants is contingent upon the presence of nkat-1 (Lemieux et al. 2015). Mimicking the pharyngeal pumping phenotype of nkat-1;kmo-1 double mutants, aat-1;kmo-1 double mutants had hyperactive pumping rates (Fig. 1C; Lemieux et al. 2015). Finally, metabolite add-back experiments showed that KynA, but not Kyn supplementation during the fasting period blocks the postfast hyperactive feeding of aat-1 mutants, supporting the notion that AAT-1 mediates Kyn transport to sites of KynA generation (Supplemental Fig. S3).

\section{aat-1 mutants have enhanced learning capacity}

KynA deficiency enhances NMDAR-dependent learning capacity in C. elegans whereas KynA accumulation has detrimental effects (Vohra et al. 2017, 2018). As in the case of pumping rate, KynA exerts its effects on learning through modulation of the activity of specific NMDARexpressing neurons. Production of KynA by the RIM neurons and regulation of NMDAR activity of the RIM neurons are sufficient to account for the effects of KynA on learning (Vohra et al. 2017). Using the butanone association assay, which has commonly been used to assess NMDAR-dependent learning (Kauffman et al. 2010), we 
found that aat-1 mutants had elevated learning capacity and could fully bypass the learning deficits of kmo-1 mutants (Fig. 2A,B). As in nkat-1 mutants, the enhanced learning of aat-1 mutants was dependent on the presence of NMDARs (Fig. 2B). We found previously that lengthening the training period eventually results in equally enhanced learning capacity in wild-type and KynA-deficient animals. Similarly, compared with wild-type animals, aat-1 mutants exhibited elevated learning capacity when the training period was between 30 and $50 \mathrm{~min}$; however, longer training periods resulted in elevation of learning capacity to the same extent in aat-1 mutant and wild-type animals (Fig. 2A).

Next, we monitored calcium transients in the RIM neurons by using the calcium-sensitive fluorophore GCaMP3. These GCaMP3 recordings were not in response to any stimuli but a measure of spontaneous activity of RIM neurons (Vohra et al. 2017). Similar to KynA-deficient animals (Vohra et al. 2017), aat-1 mutants had significantly longer bursts as well as increased $\mathrm{Ca}^{2+}$ transient peak values (Fig. 2C). The total integrated change of fluorescence intensity in RIM neurons was significantly greater in aat-1 mutants compared with wild-type animals (Fig. 2D). Thus, loss of aat-1 mimics the effects of reduced KynA on the RIM Ca ${ }^{2+}$ transient and learning behavior.

\section{Reconstitution of aat-1 in only the RIM neurons is} sufficient to restore wild-type learning and feeding phenotypes

We previously showed that within the nervous system nkat-1 is expressed in only a subset of neurons, specifi-

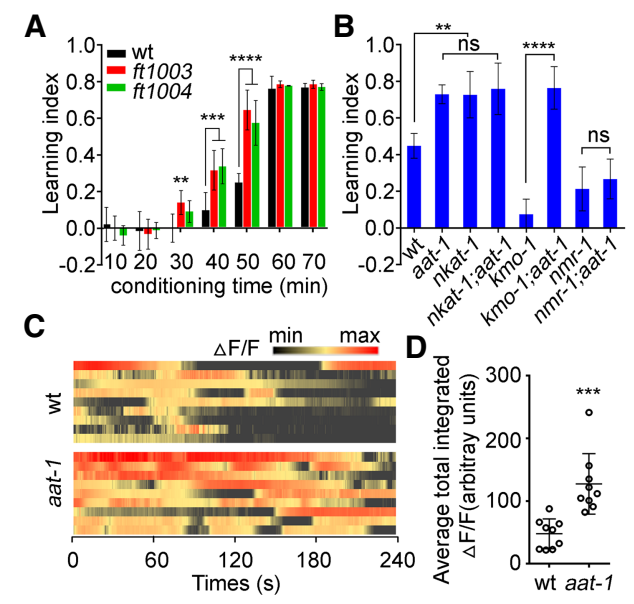

Figure 2. Loss of aat-1 increases learning and the activity of RIM neurons. (A) Effects of various durations of conditioning on the learning indices of wild-type and aat-1 mutants. $n=3$ for per condition. $\left(^{* *}\right) P<0.01 ;\left(^{* * *}\right) P<0.001 ;$ (***) $^{* *} P<0.0001$ by ANOVA (Dunnett). (B) Learning indices of the indicated strains. $n=5$ for each condition. (ns) $P>0.05$; (**) $P<0.01$; $(* * *) ~ P<0.0001$ by ANOVA (Tukey). In $A$ and $B, 100-200$ animals were used in each replicate of each genotype or condition. (C) The integrated change of GCaMP3 fluorescence intensity $(\Delta \mathrm{F} / \mathrm{F})$ in the RIM neurons over the entire 240-sec imaging window. Data from nine representative young adult animals coexpressing cex-1p::GCaMP3 and tdc-1p:: $m$ Cherry are shown as a heat map for each genotype. $(D)$ The total integrated change in fluorescence intensity over the 240 -sec imaging window as shown in $C . n=9$ animals per condition. $\left(^{* * *}\right) P<0.001$ by Student's $t$-test. cally RMDV, RIM and RID, and that KynA production from the RIM neurons is sufficient for wild-type feeding and learning phenotypes (Lemieux et al. 2015; Vohra et al. 2017). To study the relationship between sites of aat-1 expression and KynA production, we first examined the expression pattern of an mCherry reporter controlled by the putative aat-1 promoter. We consistently observed mCherry fluorescence in several pairs of head neurons, pharynx, reproductive system, excretory cells and weak transgene expression in the intestine of adult animals (Fig. 3A). Reporter fusions controlled by the aat-1 and nkat-1 promoters showed overlapping expression patterns in RMDV and RIM neurons (Fig. 3B).

Reconstitution of aat-1 cDNA using the putative aat-1 promoter or selective expression in nervous system using unc-119, a pan-neuronal promoter, completely restored wild-type feeding rate and learning capacity to aat-1 mutants (Fig. 3C,D; Supplemental Fig. S4). Similar results were found when aat-1 was selectively reconstituted in only the RIM and RIC interneurons using a $t d c$ - 1 promoter, or in only the RIM neurons using a cex-1 promoter (Fig. 3C,D; Supplemental Fig. S4). In contrast, placing the same aat-1 cDNA under the nkat-3 promoter, which expresses in the pharyngeal muscle and several head and tail cells that are different than those that express nkat-1 (Lemieux et al. 2015), failed to rescue aat-1's mutant phenotypes (Fig. 3C,D; Supplemental Fig. S4). This is consistent with our previous finding that nkat-1 mutants expressing nkat-3p::nkat-1 continue to exhibit hyperactive feeding (Lemieux et al. 2015). Thus, the anatomical site of aat-1 expression that restores normal learning and feeding to aat-1 mutants is the same interneurons, RIM, previously found as the site of KynA regulation relevant to learning and feeding (Lemieux et al. 2015; Vohra et al. 2017).

\section{AAT-1 transports kynurenine when coexpressed with ATGP-2}

Among the nine AATs in C. elegans, AAT-1, AAT-2, and AAT-3 are most homologous to human LAT1 (Veljkovic et al. 2004). Both AAT-1 and LAT1 contain conserved cysteine residues, which in the case of human LAT1 allow for covalent linkages to glycoprotein subunits 4F2HC/ SLC3A2 resulting in heterodimeric transporters (Pfeiffer et al. 1998; Veljkovic et al. 2004). Veljkovic et al. (2004) showed that AAT-1 can transport large neutral amino acids including phenylalanine and tyrosine into Xenopus laevis oocytes when coexpressed with ATGP-2, the C. elegans counterpart of heteromeric amino acid transporter glycoprotein. Plasma membrane localization of AAT-1 is also dependent on ATGP-2 (Veljkovic et al. 2004). As in aat-1 mutants, loss-of-function atgp-2 mutants displayed an increased feeding rate and enhanced learning capacity (Supplemental Fig. S5). We generated AAT-1 and ATGP2-coexpressing $X$. laevis oocytes and examined their uptake capacity of labeled $\left[{ }^{3} \mathrm{H}\right]$-L-kynurenine and large amino acid control $\left[{ }^{3} \mathrm{H}\right]$-L-phenylalanine (Fig. 4). Oocytes injected with both AAT-1 and ATGP-2 cRNAs exhibited significantly increased uptake of both of kynurenine and control phenylalanine compared with saline-injected or only ATGP-2 cRNA-injected oocytes (Fig. 4C). Time-course experiments showed that the uptake rate of kynurenine was in the linear range at $15 \mathrm{~min}$ and began to plateau at $30 \mathrm{~min}$ (Fig. 4A), so we conducted kinetic studies of AAT-1-mediated-kynurenine uptake in a range 


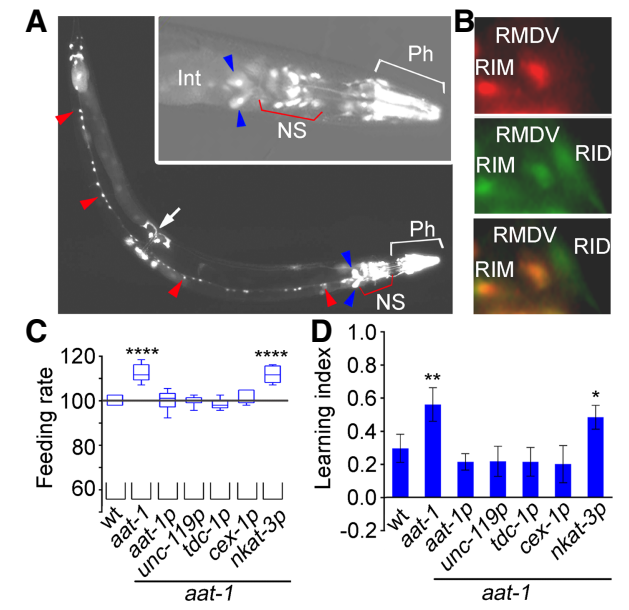

Figure 3. Localized expression of AAT-1 in nervous system regulates feeding and learning. (A) Expression of aat-1p::gfp transcriptional fusion in an adult animal. Fluorescence is seen in the pharynx $(\mathrm{Ph})$; nervous system (NS; red arrowheads, and red brackets in the inset); reproductive system (white arrow), including vulval muscles, uterine muscles, and uterine-vulva cells; excretory system (blue arrowheads); and intestine (Int). (Inset) Overlay of DIC and fluorescence images of the head region from the same animal. $(B)$ Overlapping expression patterns of aat-1p::mCherry and nkat-1p::gfp in the RIM and RMDV neurons but not RID neurons. $(C)$ Effects of reconstituting aat-1 cDNA using various promoters on pharyngeal pumping rates of aat -1 mutants. $n=9-12$ per condition. $\left(^{* * *}\right) P<0.0001$ by ANOVA (Dunnett) in comparison with wild-type animals. $(D)$ Learning indices of the indicated strains. $n=4-7$ per condition. $(* *) P<0.01 ;(*) P<0.05$ by ANOVA (Dunnett) in comparison with wild-type animals. All transgenes are high copy extrachromosomal arrays. One-hundred to 200 animals were used in each replicate of each genotype.

of kynurenine concentrations $(0-100 \mu \mathrm{M})$ at $15 \mathrm{~min}$ (Fig. 4B). Using nonlinear regression, we estimated the $K_{M}$ and $V_{\max }$ values of $C$. elegans AAT-1/ATGP-2 transporter to be $40.1 \pm 12.1 \mu \mathrm{M}$ and $89.1 \pm 10.8$ pmole/oocyte $/ 15 \mathrm{~min}$, respectively (Fig. 4B). The $K_{\mathrm{M}}$ of kynurenine for AAT-1/ ATGP-2 is in a similar range as the $K_{M}$ of leucine for LAT1 $(18.1 \mu \mathrm{M})$ in the $X$. laevis oocytes system (Kanai et al. 1998) and kynurenine uptake by astrocytes $\left(K_{M} ; 32\right.$ $\mu \mathrm{M})$ (Speciale et al. 1989). Finally, kynurenine transport was significantly inhibited by known substrates of this transporter as exposure to $1 \mathrm{mM}$ of L-Alanine decreased kynurenine uptake by AAT-1/ATGP-2 coexpressing oocytes (Fig. 4C). Thus, the C. elegans AAT-1 transports kynurenine in a concentration-dependent fashion, is likely a highly affinity transporter for kynurenine, and inhibited by other substrates of the transporter.

Altered kynurenine pathway metabolite levels in several neurodegenerative and neuropsychiatric disorders have raised the notion of kynurenergic manipulation as a therapeutic strategy (Erhardt et al. 2009; Stone and Darlington 2013; Kozak et al. 2014). Delivery of therapeutic agents to the brain space is considered particularly challenging (Henderson and Piquette-Miller 2015). Manipulation of kynurenine substrate availability has been proposed as one strategy for affecting brain KynA levels (Stone and Darlington 2013; Agudelo et al. 2014; Vohra et al. 2017). The existing data on LAT-1, the mammalian counterpart of AAT-1, supports the notion that these large neural amino acid transporters can indeed transport kynurenine across the blood-brain barrier (Fukui et al. 1991; Sekine et al. 2016), into astrocytes (Speciale et al. 1989), and into T-cells (Sinclair et al. 2018). Our findings here provide evidence that this transport mechanism can be targeted to affect neural KynA levels in an intact organism with the expected effects on learning capacity.

Given the expression pattern of aat-1 and that AAT-1 is capable of transporting many substrates other than Kyn, AAT-1 is likely involved in processes beyond feeding and learning. AAT-1 has been proposed to function as a cysteine/glutamate exchanger on glial cells in C. elegans (Gibson et al. 2018) and LAT-1 like transporters in Drosophila affect dopaminergic transmission and sleep regulation (Aboudhiaf et al. 2018). Furthermore, the physiological functions of AAT transporters are dependent on their heterodimeric partners. For example, AAT-1 and AAT-3 facilitate the transport of amino acids with the help of ATGP-2 but not ATGP-1 or mammalian 4F2HC (Velikovic et al. 2004). Thus, consideration of transporters as therapeutic targets must balance beneficial effects towards intended outcomes with potential effects on other biological processes regulated by the same transporter.

\section{Materials and methods}

Nematode strains and growth conditions

Strains used for this study are listed in the Supplemental Material. All mutants were backcrossed into the wild-type background at least four times. C. elegans strains were cultured at $20^{\circ} \mathrm{C}$ on NGM agar plates seeded with OP50 E. coli or HT-115 E. coli RNAi clones.
A

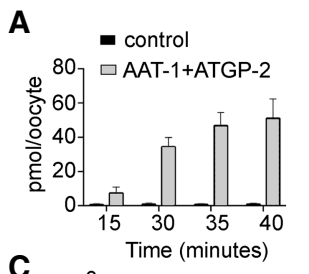

C

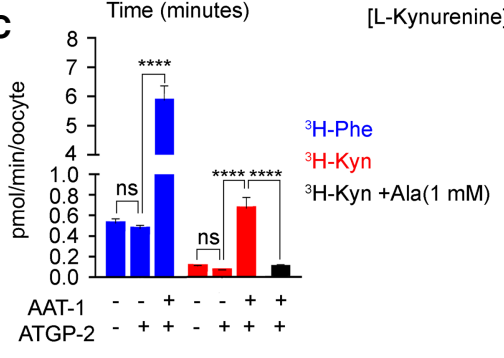

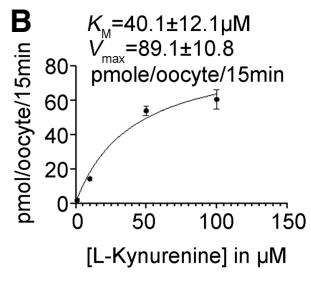

$\mathrm{H}-\mathrm{Phe}$

${ }^{3} \mathrm{H}-\mathrm{Kyn}$

M)
Figure 4. AAT-1 transports kynurenine when coexpressed with ATGP-2. (A) Time-course experiment conducted at various durations (0-40 $\mathrm{min}$ ) to determine the linear range of kynurenine transport (10 $\mu \mathrm{M})$ in Xenopus oocytes coexpressing the C. elegans AAT-1 and ATGP-2. Control oocytes were injected with saline. (B) Based on the time course results, kinetic experiments were 15 min long. Experiments were conducted by incubating Xenopus oocytes coexpressing C. elegans AAT-1 and ATGP-2 with increasing concentrations of Lkynurenine $(1 \mu \mathrm{M}, 10 \mu \mathrm{M}, 50 \mu \mathrm{M}$, and $100 \mu \mathrm{M})$. Rate of uptake versus concentration was plotted; $K_{M}$ and $V_{\max }$ values were estimated by fitting the curves to a Michaelis-Menten equation using Graphpad Prism software. $(C)$ Uptake of L-phenylalanine (10 $\mu \mathrm{M}, 3 \mathrm{~min}$; positive control) or L-kynurenine (10 $\mu \mathrm{M}, 15 \mathrm{~min})$ in Xenopus oocytes coexpressing AAT-1 and ATGP-2 was significantly higher than uptake in oocytes expressing ATGP-2 alone or in saline-injected oocytes. The uptake of kynurenine was significantly inhibited by L-alanine (1 $\mathrm{mM}$ ), a known substrate of C. elegans AAT-1 and ATGP-2 transporters (Veljkovic et al. 2004). Graphs showing radiolabeled substrate uptake (Fig. 4A) and uptake rates (Fig. 4B,C). $n=5-10$ oocytes per condition. (ns) $P>0.05 ;\left({ }^{* * *}\right) P<0.0001$ by ANOVA (Tukey). 


\section{Radiolabeled substrate uptake assays in X. laevis oocytes}

All capped RNA (cRNA) was synthesized using the mMessage mMachine SP6 kit (Ambion) and injected into oocytes by Ecocyte Bio Science LLC. Oocytes were incubated at $18^{\circ} \mathrm{C}$ in modified Barth's solution with gentamicin that was replenished every $24 \mathrm{~h}$ followed by three washes and preincubation for at least $5 \mathrm{~min}$ at $25^{\circ} \mathrm{C}$ in uptake buffer. For uptake assays, sodium-free modified Barth's solution containing labeled $\left[{ }^{3} \mathrm{H}\right]$-L-phenylalanine or $\left[{ }^{3} \mathrm{H}\right]$-L-kynurenine (PerkinElmer) was used. Oocytes were then washed five times in ice-cold uptake buffer and lysed in $0.5 \mathrm{~mL}$ of $1 \%$ SDS solution for at least $1 \mathrm{~h}$. Radioactivity (disintegrations per minute) was measured using a liquid scintillation counter machine. A detailed procedure is described in Supplemental Material.

\section{Pharyngeal pumping assay}

Pharyngeal pumping rates were measured on day 1 gravid adults by counting the contraction of the pharyngeal bulb over a 10-sec period as described before (Lemieux et al. 2015). Source data for the pharyngeal pumping experiments are listed in Supplemental Table S1.

\section{Learning assay}

Short-term butanone associative learning was performed as previously described (Kauffman et al. 2010). Unless otherwise noted, 100-200 synchronized day 1 adult animals grown on 6-cm NGM plate were trained for 50 min with $2 \mu \mathrm{L}$ of $10 \%$ butanone on lid, and then tested for butanone chemotaxis at $20^{\circ} \mathrm{C}$. Additional procedure details are described in the Supplemental Material. Source data are available in Supplemental Table S2.

\section{Spontaneous $\mathrm{Ca}^{2+}$ transients}

Day 1 adult animals that express Ex[cex-1p::GCaMP3; $t d c-1 p:: m$ Cherry] were mounted on a $10 \%$ agarose pad containing $2 \mu \mathrm{L}$ of $0.1 \mathrm{~mm}$ polystyrene beads followed by the immobilization with cover slips, and GCaMP3 imaging of the RIM neurons were performed as described before (Lemieux et al. 2015). Additional procedure details are available in the Supplemental Material.

\section{Statistics}

Data were analyzed using GraphPad Prism 6.0 software package and are provided as mean \pm standard deviation unless otherwise noted. Box and whisker plots were used to present all the feeding data. Boxes represent 25 th to the 75 th percentile of the data, with an additional line showing 50th percentile, and the whiskers show the 5th and 95th percentiles. Two-tailed student's $t$-test were used when comparing two conditions. For multiple comparison test, one or two-way ANOVA was performed. Studies were not blinded but various strains were examined at different times by different individuals.

\section{Acknowledgments}

We thank Dr. Masako Asahina for the suggestion of generating null mutants, and Dr. Daniel L Minor Jr. for allowing the generous use of his oocyte incubator. Some C. elegans strains were provided by the Caenorhabditis Genetics Center, funded by National Institutes of Health (NIH) Office of Research Infrastructure Programs (P40 OD010440). This work was supported by funds from the NIH (R01 AG046400) and the Program in Breakthrough Biomedical Research to K.A. Additional support was provided by a grant to the University of California at San Francisco from the Howard Hughes Medical Institute through the James H. Gilliam Fellowships for Advanced Study Program (O.J.E.).

Author contributions: L.L., G.A.L., O.J.E., K.M.G., and K.A. developed and designed the experiments. L.L., G.A.L., and O.J.E. performed the experiments and performed data analyses. K.M.G. and K.A. supervised the project. L.L., O.J.E., and K.A wrote the paper with input from all coauthors.

\section{References}

Aboudhiaf S, Alves G, Parrot S, Amri M, Simonnet MM, Grosjean Y, Manière $\mathrm{G}$, Seugnet L. 2018. LAT1-like transporters regulate dopaminergic transmission and sleep in Drosophila. Sleep 41: zsy137. doi:10.1093/ sleep/zsy137

Agudelo LZ, Femenía T, Orhan F, Porsmyr-Palmertz M, Goiny M, Martinez-Redondo V, Correia JC, Izadi M, Bhat M, Schuppe-Koistinen I, et al. 2014. Skeletal muscle PGC-1 $a 1$ modulates kynurenine metabolism and mediates resilience to stress-induced depression. Cell 159: 33-45. doi:10.1016/j.cell.2014.07.051

Avery L, Horvitz HR. 1990. Effects of starvation and neuroactive drugs on feeding in Caenorhabditis elegans. J Exp Zool 253: 263-270. doi:10 $.1002 /$ jez.1402530305

Cervenka I, Agudelo LZ, Ruas JL. 2017. Kynurenines: tryptophan's metabolites in exercise, inflammation, and mental health. Science 357: eaaf9794. doi:10.1126/science.aaf9794

Cunningham KA, Hua Z, Srinivasan S, Liu J, Lee BH, Edwards RH, Ashrafi K. 2012. AMP-activated kinase links serotonergic signaling to glutamate release for regulation of feeding behavior in C. elegans. Cell Metab 16: 113-121. doi:10.1016/j.cmet.2012.05.014

Cunningham KA, Bouagnon AD, Barros AG, Lin L, Malard L, RomanoSilva MA, Ashrafi K. 2014. Loss of a neural AMP-activated kinase mimics the effects of elevated serotonin on fat, movement, and hormonal secretions. PLoS Genet 10: e1004394. doi:10.1371/journal .pgen.1004394

Erhardt S, Blennow K, Nordin C, Skogh E, Lindström LH, Engberg G. 2001. Kynurenic acid levels are elevated in the cerebrospinal fluid of patients with schizophrenia. Neurosci Lett 313: 96-98. doi:10.1016/s0304-3940 (01)02242-x

Erhardt S, Olsson SK, Engberg G. 2009. Pharmacological manipulation of kynurenic acid: potential in the treatment of psychiatric disorders. CNS Drugs 23: 91-101. doi:10.2165/00023210-200923020-00001

Fukui S, Schwarcz R, Rapoport SI, Takada Y, Smith QR. 1991. Blood-brain barrier transport of kynurenines: implications for brain synthesis and metabolism. I Neurochem 56: 2007-2017. doi:10.1111/j.1471-4159 .1991.tb03460.x

Gál EM, Sherman AD. 1980. L-kynurenine: its synthesis and possible regulatory function in brain. Neurochem Res 5: 223-239. doi:10.1007/ BF00964611

Gibson CL, Balbona JT, Niedzwiecki A, Rodriguez P, Nguyen KCQ, Hall DH, Blakely RD. 2018. Glial loss of the metallo $\beta$-lactamase domain containing protein, SWIP-10, induces age- and glutamate-signaling dependent, dopamine neuron degeneration. PLoS Genet 14: e1007269. doi:10.1371/journal.pgen.1007269

Guidetti P, Hoffman GE, Melendez-Ferro M, Albuquerque EX, Schwarcz R. 2007. Astrocytic localization of kynurenine aminotransferase II in the rat brain visualized by immunocytochemistry. Glia 55: 78-92. doi:10.1002/glia.20432

Henderson JT, Piquette-Miller M. 2015. Blood-brain barrier: an impediment to neuropharmaceuticals. Clin Pharmacol Ther 97: 308-313. doi:10.1002/cpt.77

Horvitz HR, Chalfie M, Trent C, Sulston JE, Evans PD. 1982. Serotonin and octopamine in the nematode Caenorhabditis elegans. Science 216: 1012-1014. doi:10.1126/science.6805073

Kanai Y, Segawa H, Miyamoto K, Uchino H, Takeda E, Endou H. 1998. Expression cloning and characterization of a transporter for large neutral amino acids activated by the heavy chain of 4F2 antigen (CD98). I Biol Chem 273: 23629-23632. doi:10.1074/jbc.273.37.23629

Kano T, Brockie PJ, Sassa T, Fujimoto H, Kawahara Y, Iino Y, Mellem JE, Madsen DM, Hosono R, Maricq AV. 2008. Memory in Caenorhabditis elegans is mediated by NMDA-type ionotropic glutamate receptors. Curr Biol 18: 1010-1015. doi:10.1016/j.cub.2008.05.051

Kauffman AL, Ashraf JM, Corces-Zimmerman MR, Landis JN, Murphy CT. 2010. Insulin signaling and dietary restriction differentially influence the decline of learning and memory with age. PLOS Biol 8: e1000372. doi:10.1371/journal.pbio.1000372

Kepplinger B, Baran H, Kainz A, Ferraz-Leite H, Newcombe J, Kalina P. 2005. Age-related increase of kynurenic acid in human cerebrospinal fluid-IgG and $\beta 2$-microglobulin changes. Neurosignals 14: 126-135. doi:10.1159/000086295

Kozak R, Campbell BM, Strick CA, Horner W, Hoffmann WE, Kiss T, Chapin DS, McGinnis D, Abbott AL, Roberts BM, et al. 2014. Reduction of 
Lin et al.

brain kynurenic acid improves cognitive function. I Neurosci 34: 10592-10602. doi:10.1523/JNEUROSCI.1107-14.2014

Lemieux GA, Cunningham KA, Lin L, Mayer F, Werb Z, Ashrafi K. 2015. Kynurenic acid is a nutritional cue that enables behavioral plasticity. Cell 160: 119-131. doi:10.1016/j.cell.2014.12.028

Moroni F, Russi P, Carlá V, Lombardi G. 1988. Kynurenic acid is present in the rat brain and its content increases during development and aging processes. Neurosci Lett 94: 145-150. doi:10.1016/0304-3940(88) 90285-6

Morris RG, Anderson E, Lynch GS, Baudry M. 1986. Selective impairment of learning and blockade of long-term potentiation by an N-methyl-Daspartate receptor antagonist, AP5. Nature 319: 774-776. doi:10.1038/ 319774a0

O'Farrell K, Harkin A. 2017. Stress-related regulation of the kynurenine pathway: relevance to neuropsychiatric and degenerative disorders. Neuropharmacology 112: 307-323. doi:10.1016/j.neuropharm.2015 .12 .004

Perkins MN, Stone TW. 1982. An iontophoretic investigation of the actions of convulsant kynurenines and their interaction with the endogenous excitant quinolinic acid. Brain Res 247: 184-187. doi:10.1016/ 0006-8993(82)91048-4

Pfeiffer R, Spindler B, Loffing J, Skelly PJ, Shoemaker CB, Verrey F. 1998. Functional heterodimeric amino acid transporters lacking cysteine residues involved in disulfide bond. FEBS Lett 439: 157-162. doi:10 $.1016 / \mathrm{s} 0014-5793(98)$ |01359-3

Pocivavsek A, Wu HQ, Potter MC, Elmer GI, Pellicciari R, Schwarcz R. 2011. Fluctuations in endogenous kynurenic acid control hippocampal glutamate and memory. Neuropsychopharmacology 36: 2357-2367. doi:10.1038/npp.2011.127

Potter MC, Elmer GI, Bergeron R, Albuquerque EX, Guidetti P, Wu HQ, Schwarcz R. 2010. Reduction of endogenous kynurenic acid formation enhances extracellular glutamate, hippocampal plasticity, and cognitive behavior. Neuropsychopharmacology 35: 1734-1742. doi:10 $.1038 / \mathrm{npp} .2010 .39$

Schwarcz R, Rassoulpour A, Wu HQ, Medoff D, Tamminga CA, Roberts RC. 2001. Increased cortical kynurenate content in schizophrenia. Biol Psychiatry 50: 521-530. doi:10.1016/s0006-3223(01)01078-2
Schwarcz R, Bruno JP, Muchowski PJ, Wu HQ. 2012. Kynurenines in the mammalian brain: when physiology meets pathology. Nat Rev Neurosci 13: 465-477. doi:10.1038/nrn3257

Sekine A, Kuroki Y, Urata T, Mori N, Fukuwatari T. 2016. Inhibition of large neutral amino acid transporters suppresses kynurenic acid production via inhibition of kynurenine uptake in rodent brain. Neurochem Res 41: 2256-2266. doi:10.1007/s11064-016-1940-y

Sinclair LV, Neyens D, Ramsay G, Taylor PM, Cantrell DA. 2018. Single cell analysis of kynurenine and system L amino acid transport in T cells. Nat Commun 9: 1981. doi:10.1038/s41467-01804366-7

Speciale C, Hares K, Schwarcz R, Brookes N. 1989. High-affinity uptake of L-kynurenine by a $\mathrm{Na}^{+}$-independent transporter of neutral amino acids in astrocytes. J Neurosci 9: 2066-2072. doi:10.1523/JNEUROSCI.0906-02066.1989

Stone TW, Darlington LG. 2013. The kynurenine pathway as a therapeutic target in cognitive and neurodegenerative disorders. Br J Pharmacol 169: 1211-1227. doi:10.1111/bph.12230

Sze JY, Victor M, Loer C, Shi Y, Ruvkun G. 2000. Food and metabolic signalling defects in a Caenorhabditis elegans serotonin-synthesis mutant. Nature 403: 560-564. doi:10.1038/35000609

van der Goot AT, Zhu W, Vázquez-Manrique RP, Seinstra RI, Dettmer K, Michels H, Farina F, Krijnen J, Melki R, Buijsman RC, et al. 2012. Delaying aging and the aging-associated decline in protein homeostasis by inhibition of tryptophan degradation. Proc Natl Acad Sci 109: 14912-14917. doi:10.1073/pnas.1203083109

Veljkovic E, Stasiuk S, Skelly PJ, Shoemaker CB, Verrey F. 2004. Functional characterization of Caenorhabditis elegans heteromeric amino acid transporters. I Biol Chem 279: 7655-7662. doi:10.1074/jbc .M309528200

Vohra M, Lemieux GA, Lin L, Ashrafi K. 2017. The beneficial effects of dietary restriction on learning are distinct from its effects on longevity and mediated by depletion of a neuroinhibitory metabolite. PLOS Biol 15: e2002032. doi:10.1371/journal.pbio.2002032

Vohra M, Lemieux GA, Lin L, Ashrafi K. 2018. Kynurenic acid accumulation underlies learning and memory impairment associated with aging. Genes Dev 32: 14-19. doi:10.1101/gad.307918.117 


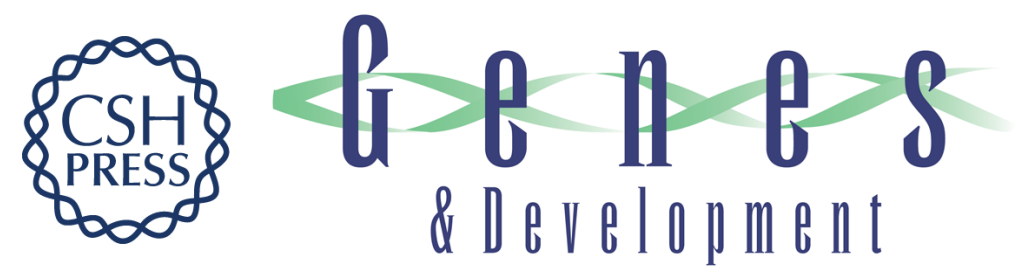

\section{Neural production of kynurenic acid in Caenorhabditis elegans requires the AAT-1 transporter}

Lin Lin, George A. Lemieux, Osatohanmwen Jessica Enogieru, et al.

Genes Dev. 2020, 34: originally published online July 16, 2020

Access the most recent version at doi:10.1101/gad.339119.120

\section{Supplemental http://genesdev.cshlp.org/content/suppl/2020/07/14/gad.339119.120.DC1 Material}

References This article cites 38 articles, 8 of which can be accessed free at: http://genesdev.cshlp.org/content/34/15-16/1033.full.html\#ref-list-1

Creative This article is distributed exclusively by Cold Spring Harbor Laboratory Press for the first Commons six months after the full-issue publication date (see

License http://genesdev.cshlp.org/site/misc/terms.xhtml). After six months, it is available under a Creative Commons License (Attribution-NonCommercial 4.0 International), as described at http://creativecommons.org/licenses/by-nc/4.0/.

Email Alerting Receive free email alerts when new articles cite this article - sign up in the box at the top Service right corner of the article or click here.

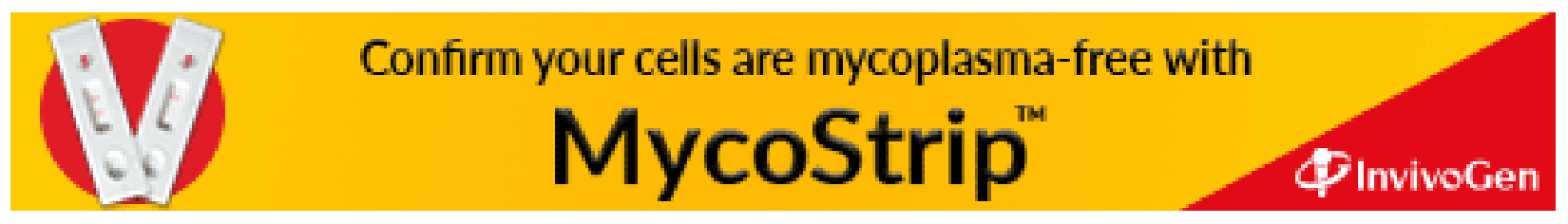

